REVISIÓN

\title{
Bacillus cereus y su papel en las intoxicaciones alimentarias
}

\author{
Bacillus cereus and food poisoning
}

\section{MsC. I rasema Pérez Portuondo}

Centro de Estudios de Biotecnología Industrial. Universidad de Oriente. Santiago de Cuba, Cuba.

\section{RESUMEN}

Introducción: Bacillus cereus es una bacteria Gram positiva, habitante frecuente de un amplio número de ambientes. Esta bacteria es la responsable del síndrome emético y del diarréico, y además se ha identificado vinculada a otras enfermedades como endocarditis, endoftalmitis, por lo que se le reconoce su carácter de patógeno oportunista.

Objetivo: revisar información sobre las toxinas producidas por B. cereus y cuáles y cómo están relacionadas con las intoxicaciones alimentarias, así como abordar otros factores responsables de la virulencia de esta bacteria.

Fuente de datos: para recopilar la información necesaria se utilizó el acceso a Internet mediante los buscadores Google y Scirus. Los artículos que se examinaron incluyeron revisiones del tema, artículos de investigación y capítulos de libros extraídos como acceso libre o solicitados a los autores.

Síntesis de los datos: se profundizó en el conocimiento sobre la toxina emética y la diarreica, la sintomatología clínica, el tiempo de aparición, así como los alimentos involucrados en cada síndrome, lo cual sirve de ayuda al clínico para determinar las causas de la toxoinfección. Se hace un bosquejo general de la microbiología de esta bacteria y otros factores que explican su virulencia y otras implicaciones en la salud humana.

Conclusiones: El conocimiento obtenido permite reconocer a B. cereus como una bacteria con importancia clínica, lo que evita considerarla solo como un contaminante ambiental.

Palabras clave: Bacillus cereus, intoxicación alimentaria, cereulida, enterotoxina, toxinas. 


\section{ABSTRACT}

Introduction: Gram positive bacterium Bacillus cereus is widely distributed in the environment. This bacterium is responsible for two food poisoning syndromes (emetic and diarrheal) and also it has been related to other diseases such as endocarditis and endophthalmitis; therefore it is recognized as opportunist pathogen.

Objective: to review the information about Bacillus cereus toxins and its connection with food poisoning, as well as to address others factors influencing this bacterium's virulence.

Data sources: for collecting this information, search engines such as Google and Scirus. Reviewed articles included research articles, systematic reviews on this topic and free accessed book chapters, or requested from the authors.

Data synthesis: the review delved into Knowledge on diarrheal and emetic toxins, clinical symptomatology and time of occurrence, as soon as foodstuffs involved in each syndrome. This helps the clinician to look for the causes of toxin infections. A general overview of Bacillus cereus microbiology and others factors that explain its virulence and other implications in the human health was given.

Conclusions: the acquired knowledge shows that Bacillus cereus is one bacterium with clinical importance rather than a mere environmental pollutant.

Key words: Bacillus cereus, food poisoning, cereulide, enterotoxin, toxins.

\section{NTRODUCCI ÓN}

Bacillus cereus es una bacteria ubicua formadora de esporas que ha sido vinculada con algunos aspectos beneficiosos y nocivos para la actividad económica de la sociedad. Esta bacteria es frecuentemente encontrada como saprofita en el suelo, agua, vegetación y aire, desde los cuales se transfiere muy fácilmente a los alimentos. La colonización de diferentes nichos ecológicos es posible debido a su buena adaptabilidad y resistencia a variadas influencias. $\mathrm{B}$. cereus produce endosporas que sobreviven a la pasteurización y son resistentes a varios desinfectantes. Además produce enzimas como lipasas, proteasas, xilanasas y otras. En la leche y productos lácteos descompone la caseína a péptidos y aminoácidos y la grasa de la leche a ácidos grasos libres, los cuales descomponen la leche y acortan su vida útil. Es propósito de este artículo, revisar información sobre las toxinas producidas por $\mathrm{B}$. cereus y cuáles y cómo están relacionadas con las intoxicaciones alimentarias, así como abordar otros factores responsables de la virulencia de esta bacteria.

\section{FUENTE DE DATOS}

La información revisada para conocer sobre esta bacteria provino del uso de buscadores como Google (http://www.google.com.cu/) y virus (http://www.scirus.com/). Se utilizaron la palabras claves Bacillus cereus y

http://scielo.sld.cu 
toxoinfecciones, foodborne and Bacillus cereus, Foodborne illness, Bacillus cereus toxins, enterotoxins of Bacillus cereus. Los artículos revisados incluyeron compendios especializados sobre el tema, así como artículos de investigación en acceso libre de los buscadores o solicitados directamente a los autores sino fue posible el acceso libre.

\section{SÍ NTESI S DE LOS DATOS}

\section{Aspectos microbiológicos}

B. cereus es una bacteria perteneciente al género Bacillus. Vista al microscopio, es un bastón alargado, gram positivo que es mótil por medio de flagelos perítricos. Las células son de 1,0-1,2 $\mu \mathrm{m}$ en el diámetro x 3,0-5,0 $\mu \mathrm{m}$ de largo. Una endospora simple puede formarse en posición central o paracentral sin hinchar el esporangio. El organismo esporula libremente en muchos medios bajo condiciones bien aireadas, aunque las células vegetativas pueden crecer anaeróbicamente. B. cereus es capaz de utilizar glucosa, fructuosa y trehalosa pero no las pentosas ni muchos azúcares alcoholes. La mayoría de las cepas hidroliza activamente el almidón, la caseína y la gelatina.

El crecimiento y la multiplicación de las células vegetativas ocurren típicamente dentro del rango de temperaturas de $10-48{ }^{\circ} \mathrm{C}$, mientras que el óptimo se encuentra entre $28-35{ }^{\circ} \mathrm{C}$. Sin embargo, se han identificado variantes psicrotróficas de $B$. cereus en leche cruda y pasteurizada capaces de crecer e iniciar la descomposición a temperaturas tan bajas como $5{ }^{\circ} \mathrm{C}$. El pH óptimo de crecimiento se encuentra entre 4,3 y 9,3. El rango mínimo de actividad del agua para el crecimiento vegetativo es de 0,912-0,950. Su tiempo de generación en medio artificial a $30{ }^{\circ} \mathrm{C}$ es de $18-27 \mathrm{~min}$. Cuando carecen de nutrientes esporulan, lo cual le permite sobrevivir largos períodos de carencia nutricional. Las esporas resisten una serie de factores ambientales como altas temperaturas $\left(D_{100}{ }^{\circ} \mathrm{C}\right.$ en leche descremada hasta $3 \mathrm{~min}, \mathrm{D}_{121}{ }^{\circ} \mathrm{C}$ en aceite vegetal hasta $30 \mathrm{~min}, \mathrm{D}_{95}{ }^{\circ} \mathrm{C}$ en agua destilada de 1,5-36 $\mathrm{min}$ ), radiaciones y reactivos químicos. ${ }^{1,2}$

La diferenciación entre B. cereus y las especies relacionadas es complicada por la similitud en las propiedades fenotípicas y genotípicas. Se ha registrado más del 99 $\%$ de similitud de la secuencia de la estructura primaria del RNAr16S entre B. cereus, Bacillus anthracis, Bacillus mycoides y Bacillus thuringiensis. Existen algunas características distintivas entre estas especies tan relacionadas. $B$. anthracis tiene dos plásmidos de virulencia, B. thuringiensis produce deltaendotoxinas en forma de inclusiones cristalinas parasporales y las colonias de B. cereus variedad mycoides muestran un crecimiento rizoide en agar sangre. Basados en la similitud existente entre ellas en su genoma, todas se han considerado variedades de $B$. cereus. ${ }^{3}$

Existen muchas razones que pueden explicar los problemas causados por B. cereus. Este es un microorganismo ubicuo en el medio ambiente y que puede contaminar fácilmente cualquier sistema de producción y procesamiento de alimentos. Debido a la formación de endosporas, esta bacteria puede sobrevivir a la pasteurización y el calentamiento incluso a la irradiación con rayos gamma, usada para reducir los patógenos en los alimentos. ${ }^{4}$ 


\section{Incidencia en la salud alimentaria}

El envenenamiento alimentario por B. cereus se describió por primera vez en 1950 luego del consumo de salsa de vainilla contaminada. Además de este estudio temprano esta bacteria se ha identificado en otros brotes, muchos de los cuales no fueron notificados debido a que sus síntomas generalmente son leves, autolimitados o se confunden con los de otros patógenos. ${ }^{4,5}$

El envenenamiento alimentario se define como cualquier enfermedad de naturaleza tóxica o infecciosa causada por el consumo de alimentos o líquidos. El término es habitualmente usado para describir la enfermedad, usualmente vómitos o diarreas causadas por bacterias, virus o parásitos. ${ }^{6}$ Se ha demostrado que este microorganismo produce siete tipos de toxinas: la cereulida (toxina emética), la cual ha sido ampliamente caracterizada en los últimos años, ${ }^{7}$ tres enterotoxinas (hemolisina BL o HBL, no-hemolítica o NHE y enterotoxina T o EntT), ${ }^{8}$ las cuales lo hacen responsable de dos síndromes, el síndrome emético y el diarreico, además de tres fosfolipasas. ${ }^{5}$

La cantidad de expresión de toxinas de B. cereus depende del medio de cultivo, así como de factores como el pH, aireación y presencia y concentración de ciertos carbohidratos..$^{5,9}$

El síndrome emético está caracterizado por náuseas agudas y vómitos similares a los producidos por intoxicación con Staphylococcus aureus. Estos síntomas se desarrollan notablemente entre 1-5 h después del consumo del alimento contaminado conteniendo la toxina preformada. La toxina es un dodecadepsipéptido llamado cereulida que provoca emesis al estimular la vía vago aferente a través de su unión con el receptor de la serotonina. La producción de la toxina ocurre durante la fase estacionaria del crecimiento del microorganismo, ${ }^{4,7}$ y se acumula en el alimento a medida que transcurre el tiempo. La estructura de la toxina explica su resistencia a los métodos de tratamiento de los alimentos y el que esté preformada al ingerirse da lugar a la rapidez con que aparecen los síntomas.

La forma diarregénica de la intoxicación con B. cereus se desencadena por la acción de al menos dos tipos de enterotoxinas de tres componentes (HBL y NHE) producidas durante la fase exponencial del crecimiento vegetativo de la bacteria, en el intestino delgado del huésped. ${ }^{10}$ Se considera que ninguna de las tres proteínas que forma cada enterotoxina (una de unión y dos factores líticos) es capaz de atravesar intacta la barrera del estómago. Esto significa que las enterotoxinas preformadas o extracelulares en el alimento no juegan ningún papel en la patogénesis de $B$. cereus. Incluso, se considera generalmente que las células vegetativas no sobreviven en el $\mathrm{pH}$ tan bajo del estómago pero las esporas sí. Por lo que se cree que la ruta del síndrome diarreico es la germinación de la espora, el crecimiento y la producción simultánea de la enterotoxina.

No obstante, Dressman y otros, ${ }^{11}$ plantean que la toma de alimento influye en el $\mathrm{pH}$ del estómago, el cual no es constantemente bajo. Las investigaciones muestran que en dependencia del tipo de alimento y la edad de los individuos el $\mathrm{pH}$ puede, al menos temporalmente, alcanzar valores altos. Lo anterior permitiría la sobrevivencia de células vegetativas, lo que conllevaría a replantear la ruta de producción de este síndrome. 
La diarrea causada por B. cereus es del tipo secretor, similar a la producida por Vibrio cholera. Esta diarrea secretora se caracteriza por una perturbación del movimiento del agua y los electrolitos a través del epitelio del intestino delgado, más frecuentemente relacionado con las enterotoxinas, aunque se plantea que no está claro como los componentes líticos de la enterotoxina entran al citoplasma de la célula hospedera para estimular a un segundo mensajero que provoca la perturbación en el intercambio de agua y electrolitos. ${ }^{12}$ Se ha sugerido que esto ocurre porque la enterotoxina de B. cereus usa la ruta del AMPc para perturbar el transporte de electrones. ${ }^{13,14}$

Los síntomas en los organismos susceptibles ocurren en aproximadamente $12 \mathrm{~h}$ luego de la ingestión de la cepa productora de enterotoxinas junto con el alimento e incluye dolor intestinal, diarrea, cólicos similares a la intoxicación con toxinas de Clostridium perfringens. El recobrado de ambos síntomas es rápido, usualmente dentro de 12-24 h.

Se conoce que la enterotoxina se une a un receptor en el lumen intestinal, conocer cuál es el receptor permitiría buscar una sustancia que al bloquearlo evite la adherencia de dicha toxina, pero como generalmente los receptores están involucrados en múltiples funciones entonces es más lógico dirigir la atención hacia la toxina e identificar el sitio de unión al receptor y atacar este sitio, por ejemplo, con una sustancia que bloquee su adherencia.

La enterotoxina se produce en la fase exponencial tardía pero la fase vegetativa bacteriana y la toxina preformada no sobreviven al ambiente extremo que encuentran en el estómago. Por lo anterior se plantea que la espora, que se consume habitualmente con el alimento, llega al intestino delgado, germina y da lugar a la célula vegetativa la cual durante su crecimiento secreta la enterotoxina y produce su acción. Este proceso dura aproximadamente $12 \mathrm{~h}$, momento en que aparecen los síntomas. La literatura refiere que el tránsito medio de la espora por el intestino delgado es de aproximadamente $8 \mathrm{~h}$, por lo que en ese tiempo la espora debe adherirse al epitelio intestinal para no ser arrastrada por los movimientos peristálticos del intestino y expulsado del organismo. Se ha referenciado que las cepas patógenas o con capacidad enterotóxicas generan esporas con apéndices que permiten su adherencia al lumen intestinal, lo cual evitaría su arrastre. ${ }^{15}$

Los complejos de enterotoxinas pueden ser detectados aplicando inmunoensayos comerciales. El conjunto de materiales para la aglutinación pasiva reversa en látex de la enterotoxina de $B$. cereus (B. cereus Enterotoxin Reversed Passive Latex Agglutination, BCET-RPLA kit, Oxoid), detecta el componente L2 de la enterotoxina HBL y la proteína de $45 \mathrm{kDa}$ de la enterotoxina NHE es la principal proteína detectada por el inmunoensayo visual para la enterotoxina diarréica de Bacillus (Bacillus Diarrheal Enterotoxin Visual Immunoassay, BDE, Tecra). ${ }^{4}$ No obstante, la detección inmunoquímica de las enterotoxinas no es completamente satisfactoria y se necesita un amplio rango de pruebas in vivo e in vitro para estimar la toxicidad de los filtrados de los cultivos, por ejemplo, en la prueba de letalidad del ratón, la reacción de permeabilidad vascular, la prueba del lazo ileal en conejo, entre otras. A diferencia de las enterotoxinas, la toxina emética de $B$. cereus es pobremente antigénica y difícil de detectar por métodos de inmunoquímica, por lo tanto su identificación se basa en bioensayos. ${ }^{16-18}$

Los alimentos crudos de origen vegetal son la fuente principal de B. cereus. La amplia distribución del organismo, la habilidad de las esporas de sobrevivir en los alimentos deshidratados y su resistencia térmica significa, que la mayoría de los alimentos que se consumen podrían contener $B$. cereus lo cual requerirá de 
medidas de control para prevenir el crecimiento, especialmente después de la cocción que elimina la flora competidora.

Las cepas que producen la toxina emética crecen bien en platos con arroz y otros alimentos farináceos, mientras que las cepas que producen la toxina diarregénica crece en una amplia variedad de alimentos que van desde vegetales hasta salsas y guisados. Numerosas hierbas desecadas, especias para condimentar y alimentos deshidratados han mostrado presencia de esta bacteria. ${ }^{2}$ Se ha encontrado que las cepas de $\mathrm{B}$. cereus que producen la enterotoxina no producen la toxina emética, así como también que no todas las cepas de $B$. cereus pueden producir la toxina emética, solo las capaces de vivir en el arroz. El por qué de esta diferenciación, así como de la relación entre el tipo de cepa toxigénica y el alimento donde crece no se conoce, también se desconoce hasta el momento las ventajas ecológicas que pudiera aportar la toxina emética que es tan resistente y tan infrecuente. ${ }^{19,20}$

Aunque la mayoría de las cepas de B. cereus tienen la potencialidad para producir la enterotoxina, la severidad de la enfermedad dependerá de la cantidad de toxina producida, la cual puede estar relacionada con la composición del alimento, así como de la condición inmunológica del individuo afectado pues se conoce que los pacientes inmunocomprometidos son particularmente susceptibles a los enteropatógenos bacterianos. ${ }^{21}$

Que este microorganismo pueda ser encontrado en casi todos los alimentos no quiere decir que hay que evitar los alimentos usualmente implicados en estas intoxicaciones sino sencillamente hay que seguir una serie de medidas que evitarán que estos alimentos $u$ otros se conviertan en una fuente de enfermedades. ${ }^{2}$

El número de envenenamientos alimentarios causados por $B$. cereus puede que haya sido sobreestimado debido a que $B$. thuringiensis, una especie altamente relacionada con $\mathrm{B}$. cereus, ha sido aislada de brotes de gastroenteritis ${ }^{22}$ y además ha mostrado una citotoxicidad idéntica a las cepas enteroxigénicas de $B$. cereus. ${ }^{23,24}$ La discriminación entre estas dos especies no es posible usando técnicas bioquímicas comunes debido a su semejanza, lo que provoca un grave problema pues B. thuringiensis es ampliamente usado como insecticida en la agricultura. No obstante, un método rápido y sensible basado en pruebas de DNA específico ha sido desarrollado por TeGiffel y otros, ${ }^{25}$ para diferenciar estas dos especies.

Hasta aquí hemos visto los síndromes causados por esta bacteria y que involucran a los alimentos, así como las toxinas que los originan y su modo de acción, lo que permite explicar la sintomatología que exhiben. Con este conocimiento se fundamentan una vez más las medidas higiénicas a tomar para evitar las infecciones alimentarias por esta u otra bacteria como son las de cocer bien los alimentos, conservarlos adecuadamente, consumirlos preferentemente inmediatamente después de su elaboración, entre otras medidas de carácter general. Hay que tener en cuenta que el $\mathrm{B}$. cereus es una bacteria cuyo crecimiento óptimo tiene lugar entre los 28 y $35 \stackrel{\circ}{\circ}$, condiciones imperantes preferentemente en países tropicales como es el caso de Cuba, lo cual junto con los nutrientes aportados por los alimentos preparados y no consumidos inmediatamente pueden propiciar la germinación de las esporas, la rápida multiplicación de esta bacteria y con ello la formación y acumulación en el alimento de las toxinas y la aparición posterior de la enfermedad.

Aunque las infecciones alimentarias causadas por esta bacteria se resuelven generalmente en $24 \mathrm{~h}$, no por ello dejan de influir en la calidad de vida del individuo afectado y en las pérdidas económicas que tiene al conllevar ausencias laborales y gastos en atención médica. Además, puede producir afectaciones de 
importancia a individuos en edades extremas (edades tempranas y tercera edad), o depauperados por largas enfermedades debilitantes debido a la deshidratación a la que pueden conducir. Todo lo anterior hace valedero profundizar sobre esta bacteria no muy conocida como causante de enfermedades relacionadas con los alimentos y si como indicador de contaminación con tierra de locales y productos industriales.

\section{Otras implicaciones}

Además de su capacidad para provocar intoxicaciones alimentarias, B. cereus también está involucrada en infecciones locales y sistémicas, especialmente asociadas con pacientes inmunocomprometidos, neonatos, drogodependientes y pacientes con heridas quirúrgicas o traumáticas o catéteres. Las cepas aisladas de este tipo de infecciones han mostrado su habilidad para sintetizar exotoxinas necrotizantes semejantes a hemolisinas y fosfolipasas. ${ }^{26}$

Las infecciones en las heridas postoperatorias o postrauma causadas por B. cereus se asocian con la producción de un factor de permeabilidad vascular dermonecrótico, HBL. Las infecciones severas por esta bacteria no son comunes. La bacteriemia en la mayoría de los casos es transitoria y nada peligrosa pero ocasionalmente causan serias infecciones.

Las cepas de $B$. cereus aisladas de las infecciones se han mostrado sensibles a cloranfenicol, clindamicina, vancomicina, gentamicina, estreptomicina, eritromicina y son usualmente resistentes a los antibióticos $\beta$-lactámicos incluidas las cefalosporinas de 3ra. generación. La explicación se halla en que esta bacteria produce tres tipos diferentes de $\beta$-lactamasas, la $\beta$-lactamasa I o tipo A, es una penicilinasa extracelular con una serina en el sitio activo. $\beta$-lactamasa II o tipo $C$, que se activa con la unión de los iones cinc y cobalto y la III, que es una lipoproteína de clase A unida a membrana que tiene además una forma secretada. ${ }^{27-29}$

Las fosfolipasas $C$ producidas se supone que contribuyen al daño tisular por inducir la desgranulación de los neutrófilos humanos. ${ }^{30} \mathrm{~B}$. cereus produce tres tipos de fosfolipasa $C$, cada una con un mecanismo diferente: fosfatidilinositol hidrolasa (rompe el fosfatidilinositol y sus derivados glicosilados los cuales anclan proteínas de la membrana plasmática), fosfatidilcolina hidrolasa (hidroliza fosfatidilcolina, fosfatidiletanolamina y fosfatidilserina) y esfingomielinasa hemolítica (hidroliza la esfingomielina y rompe los eritrocitos). Se conoce que este amplio espectro induce la producción de una metaloproteinasa de la matriz (MMP-9 específica) en las células epiteliales humanas, ${ }^{31}$ lo que sugiere su influencia en la destrucción del epitelio de los tejidos infectados y el incremento de la destrucción de la matriz subepitelial.

La pregunta que surge en estos momentos es cómo una bacteria que se considera asociada principalmente al suelo presenta enzimas como las fosfolipasas C, específicamente la fosfoinositol fosfatidil hidrolasa, capaz de degradar compuestos que forman parte de las células eucariotas como es el fosfatidilinositol, afectar el esqueleto glicofosfolipídico y con ello la actividad de las enzimas acopladas a este; y esfingomielinasa que degrada la esfingomielina, un componente muy abundante en los eritrocitos o glóbulos rojos, pero no existente en los procariotas. Ambas enzimas se encuentran en patógenos humanos como Listeria monocytogenes y Pseudomona aeruginosa, donde actúan como factores de virulencia facilitando la infección del huésped y su supervivencia en este. ${ }^{32}$ 
Otros factores de virulencia son las ya comentadas enterotoxina y toxina emética, además de hemolisinas como cereolisina similar a la estreptolisina $0,{ }^{33}$ la hemolisina II yla hemolisina III. ${ }^{34}$

Se considera que su nicho fundamental es el suelo donde su gran diversidad metabólica le da ventaja sobre otros habitantes microbianos para obtener nutrientes. Las fosfolipasas le dan la posibilidad a las bacterias de liberar los ácidos grasos del diacilglicerol una vez separado este del esqueleto de la fosfatidilcolina, y ser usados entonces como fuente de carbono y energía y para la síntesis de otros polisacáridos. ${ }^{35}$ También le permiten acceder al fósforo como fosfato pues en conjunto con fosfatasas pueden liberar este fosfato de la fosfatidilcolina que ha quedado libre. ${ }^{36}$ B. cereus, debido a su posibilidad de producir fosfolipasas, es capaz de acceder a nutrientes como el fósforo y el carbono utilizando estas enzimas para degradar los fosfolípidos de las membranas celulares, además de acceder al contenido celular.

Lereclus y otros, ${ }^{37}$ plantean que $B$. cereus es un patógeno facultativo de insectos y aunque los mecanismos patogénicos no estaban claros en aquel momento, se asume que la habilidad para desarrollarse en ellos era debido en parte, a su alta producción de fosfolipasas. Margulis y otros, ${ }^{38}$ encuentran que las formas Arthromitus simbiontes de insectos (termites) eran formas especiales de esta bacteria. Barth e investigadores, ${ }^{39}$ ya en el 2004 comentan en su publicación que $B$. cereus presenta proteínas insecticidas vegetativas (VIP), con una estructura binaria como la del ántrax y que es letal para los gusanos que viven en las raíces del maíz.

Se sugiere que estas enzimas colaboran en este tipo de vida al permitirle acceder a los recursos energéticos encerrados en las membranas de los insectos. Accidentalmente llegan al hombre donde pudieran utilizar los mismos recursos al estar formadas sus membranas con los mismos fosfolípidos que los insectos. Todo lo anterior pudiera ser interpretado como un mecanismo de adaptación al ambiente. B. cereus es una especie que recibe mucha atención por lo que podemos aprender sobre la existencia de tipos virulentos y no virulentos. Sus características puede reflejar la naturaleza diversa de la bacteria, primariamente existiendo como saprofito del suelo con una fisiología bien adaptada para el tracto intestinal y actuando como patógeno oportunista involucrado en infecciones locales y sistémicas.

Se puede concluir que como se ha mostrado, los estudios sobre esta bacteria van cobrando interés en la medida en que se descubren más sitios de incidencia de su virulencia y aumenta el número de personas que se convierten en susceptibles de contraer las enfermedades causadas por B. cereus debido a la inmunodepresión originada por la epidemia de sida. La mayoría de los países subdesarrollados tienen un clima tropical donde las poblaciones de esta bacteria tienen altas densidades en el suelo, pero a la vez el nivel de pobreza y el bajo nivel cultural existentes hacen que coexista una insuficiente cultura sanitaria que los proteja de infecciones oportunistas de este u otros microorganismos. También se ha visto la importancia que tienen las toxinas de B. cereus como factores de virulencia. Todo lo anterior avala los esfuerzos para ampliar los conocimientos sobre esta bacteria y sus toxinas. 


\section{REFERENCI AS BI LI OGRÁFI CAS}

1. Kramer JM, Gilbert RJ. Bacillus cereus gastroenteritis. En: Tu AT, editor. Food Poisoning. Handbook of Natural Toxins. Vol 7. New York: Marcel Dekker Inc.; 1992. p. 119-53.

2. Ministry of Health. Bacillus cereus [Internet]. New York: Minister of Health [citado 22 Ene 2004]. Available from: http://healthvermont.gov/enviro/ph_lab/MicrobiologyHomePage.aspx\#TB

3. Helgason E, Økstad OA, Caugant DA, Johansen HA, Fouet A. Bacillus anthrax, Bacillus cereus, and Bacillus thuringiensis. One species on the basis of genetic evidence. App Environ Microbiol. 2000;66:2627-30.

4. Kotiranta A, Lounatmaa K, Haapasalo M. Epidemiology and pathogenesis of Bacillus cereus infections. Microb Infect. 2000;2: 189-98.

5. Mckillip JL. Prevalence and expression of enterotoxins in Bacillus cereus and other Bacillus spp., a a literature review. Antonie van Leeuwenhoek. 2000; 77: 393-9.

6. Food Poison [Internet]. [citado 21 Jul 2005]. Available from: http://www5.geometry.net/detail/health_conditions food poisoning page no $6 . \mathrm{html}$

7. Agata N, Ohta M, Mori M, Isobe M. A novel dodecadepsipeptide, cereulide, is an emetic toxin of Bacillus cereus. FEMS Microbiol Lett. 1995;129:17-20.

8. Andersen GI, Skeie M, Sørhaug T, Langsrud T, Granum PE. Growth and toxin profiles of Bacillus cereus isolated from different food sources. Int J Food Microbiol. 2001; 69: 237-46.

9. Wijnands LM, Dufrenne JB, van Leusden FM. The pathogenic mechanism of the diarrheal syndrome caused by Bacillus cereus. RIVM report [Internet]. 2001/2002 [citado 6 Ene 2006]. Available http://www.rivm.nl/bibliotheek/rapporten/250912001.pdf

10. Beecher DJ, Schoeni JL, Wong ACL. Enterotoxic activity of hemolysin BL from Bacillus cereus. Infect Immunol. 1995;63:4423-8.

11. Dressman JB, Berardi RR, Dermentzoglou LC, Russell TL, Schmaltz SP, Barnett $\mathrm{JL}$, et al. Upper gastrointestinal $(\mathrm{Gl}) \mathrm{pH}$ in young, healthy men and women. Pharmaceutical Res. 1990; 7: 756-61.

12. Belaiche J. Pathophysiology of acute infectious diarrhea. Acta Endoscopic. 2000; 30: 177-84.

13. Powell DW. Enterogenic diarrea: mechanisms and prospects for therapy. In: Dorner F, Drew J, editors. Pharmacology of bacterial toxins. Oxford: Pergamon; 1987. p. 173-83.

14. Laohachai $\mathrm{KN}$, Bahadi R, Hardo MB, Kourie JI. The role of bacterial and nonbacterial toxins in the induction of changes in membrane transport: implications for diarrhea. Toxicol. 2003; 42:687-707.

http://scielo.sld.cu 
15. Stalheim T, Granum PE. Characterization of spores appendages from Bacillus cereus strains. J App Microbiol. 2001;91:839-45.

16. Andersson MA, Mikkola R, Helin J, Andersson CM, Salkinoja-Salonen M. A novel sensitive bioassay for detection of Bacillus cereus emetic toxin and related depsipeptide ionophores. App Environ Microbiol. 1998;64: 1338-43.

17. Beattie SH, Williams AG. Detection of toxigenic strains of Bacillus cereus and other Bacillus spp. with an improved cytotoxicity assay. Lett App Microbiol. 1999; 28: 221-5.

18. Mikami T, Horikawa T, Murakami T, Matsumoto T, Yamatawa A. An improved method for detecting cytostatic toxin (emetic toxin) of Bacillus cereus ant its application to foods samples. FEMS Microbiol Lett. 1994; 119:53-8.

19. Altayar M, Sutherland AD. Bacillus cereus is common in the environment but emetic toxic producing isolates are rare. J Appl Microbiol. 2006; 100(1): 7-14.

20. Ehling Schulz $M$, Svensson B, Guinebretiere $M H$, Lindback $T$, Schultz A. Emetic toxic formation of Bacillus cereus is restricted to a single evolutionary lineage of clearly related strains. Microbiol. 2005; 151: 183-97.

21. Rowan NJ, Anderson JG. Growth and enterotoxin production by diarrhoeagenic Bacillus cereus in dietary supplements prepared for hospitalised HIV patients. J Hosp Infect. 1998; 38: 139-46.

22. Jackson SG, Goodbrand RB, Ahmed R, Kasatiya S. Bacillus cereus and Bacillus thuringiensis isolated in a gastroenteritis outbreak investigation. Lett Appl Microbiol. 1995; $21: 103-5$.

23. Hansen BM, Hendriksen NB. Detection of Enterotoxic Bacillus cereus and Bacillus thuringiensis Strains by PCR Analysis. App Environ Microbiol. 2001;67(1): 185-9.

24. Phelps RJ, McKillip J L. Enterotoxin Production in Natural I solates of Bacillaceae outside the Bacillus cereus Group. App Environ Microbiol. 2002;68(6):3147-51.

25. TeGiffel MC, Beumer RR, Klijn N, Wagendorp A, Rombouts FM. Discrimination between Bacillus cereus and Bacillus thuringiensis using specific DNA probes based on variable regions of 16S rRNA. FEMS Microbiol Lett. 1997;146:47-51.

26. Turnbull PCB, Jørgensen K, Kramer JM, GilbertRJ, Parry JM. Several clinical conditions associated with Bacillus cereus and apparent involvement of exotoxins. J Clin Pathol. 1979; 32:89-293.

27. Madgwick PJ, Waley SG. b-lactamasa I from Bacillus cereus. Biochem J. 1987; 248: 657-62.

28. Orellano EG, Girardini JE, Ceccarelli EA, Vila AJ. Spectroscopic Characterization of a bionuclear metal site in Bacillus cereus b-lactamasa II. Biochemistry. 1998; 37: 10173-80.

29. Nielsen JBK, Lampen JO. b-lactamasa III of Bacillus cereus 569: Membrane lipoprotein and secreted protein. Biochemistry. 1983;22:4652-6. 
30. Wasny TK, Mummaw N, Styrt B. Desgranulation of human neutrophils after exposure to bacterial phospholipase C. Eur J Clin Infect Dis. 1990; 9:830-2.

31. Firth JD, Putnins EE, Larjava H, Uitto VJ. Bacterial phospholipase C upregulates matrix metalloproteinase expression by cultured epithelial cells. I nfect Immunol. 1997; 65: 4931-6.

32. Heinz DW, Essen LO, William RL. Structural and mechanistic comparison of prokaryotic and eukaryotic phosphoinositide-specific phospholipases C. J Mol Biol. 1998; 275: 635-50.

33. Granum PE. Bacillus cereus and its toxins. J Appl Bacteriol Symp Suppl. 1994; 76:61S-6S.

34. Baida GE, Kuzmin NP. Mechanism of action of hemolysin III from Bacillus cereus. Biochim Biophys Acta. 1996; 1284: 122-4.

35. Vasil M. Pseudomona aeruginosa. Phospholipases and phospholipids. In: Ramos $\mathrm{J}$, Levesque R, editors. Pseudomonas. Vol 4. Molecular Biology of emerging issues. New York: Springer; 2006. p. 367.

36. Guddal PH, JohansenT, Schulstad K, Little C. Apparent phosphate retrieval system in Bacillus cereus. J Bacteriol. 1989; 171:5702-6.

37. Lereclus D, Agaisse H, Gominet M, Salamitou S, Sanchis V. Identification of a Bacillus thuringiensis gene that positively regulates transcription of the phosphatidylinositol specific phospholipase $\mathrm{C}$ gene at the onset of the stationary phase. J Microbiol. 1996; 178:2749-56.

38. Margulis L, Jorgensen JZ, Dola S, Kolchinsky R, Rainey FA, Lo S. The arthromitus stage of Bacillus cereus: Intestinal symbionts of animals. Microbiol. 1998; 95: 1236-41.

39. Barth H, Aktories K, Popoff MR, Stiles BG. Binary bacterial toxins: Biochemistry, biology and applications of common Clostridium and Bacillus proteins. Microbiol Molec Biol Rev. 2004; 171:5702-6.

Recibido: 23 de noviembre de 2009.

Aprobado: 23 de junio de 2011.

I rasema Pérez Portuondo. Patricio Lumumba s/n, Santiago de Cuba 90500. Cuba. Telef.: (63) (22) 632095. Correo electrónico: iperez@cebi.uo.edu.cu 